\title{
SUBSTRATOS E FUNGO MICORRÍZICO ARBUSCULAR EM MUDAS MICROPROPAGADAS DE BANANEIRA NA FASE DE ACLIMATAÇÃ̃ ${ }^{1}$
}

\author{
ALDO VILAR TRINDADE²; GÊLVA MARIA DE LIMA LINS ${ }^{3}$; ISABEL CRISTINA SILVA MAIA ${ }^{4}$
}

\begin{abstract}
RESUMO - Visando a avaliar diferentes substratos na formação de mudas de bananeira e seu efeito na resposta da planta à inoculação do fungo micorrízico Gigaspora margarita, foi conduzido um experimento em estufa de aclimatação da Biofábrica CAMPO - CPA/Embrapa Mandioca e Fruticultura, Cruz das Almas, Bahia. Foram testados 13 substratos, com e sem inoculação do fungo, em plântulas na fase de aclimatação. A inoculação foi realizada no momento do transplante e, após 55 dias de cultivo, obtiveram-se dados de crescimento, nutrição mineral e colonização micorrízica. O fungo micorrízico arbuscular (FMA) Gigaspora margarita colonizou intensamente e mostrou-se benéfico para o desenvolvimento das mudas de bananeira, sendo o seu efeito modulado pelo substrato de crescimento; o substrato turfa + vermiculita $+5 \%$ de esterco destacou-se entre os melhores para a formação de mudas normais e sadias, mas só quando associado à inoculação do FMA; o uso de substrato comercial Rendmax Citrus promoveu o melhor desenvolvimento das mudas, mas inibiu a colonização e o efeito da micorriza; a complementação mineral do Rendmax Citrus não se mostrou necessária para o cultivo de mudas de bananeira; o uso de vermicomposto mostrou-se promissor para a produção de mudas de bananeira, permitindo o efeito da inoculação com FMA.
\end{abstract}

Termos para indexação: Musa spp., micorriza, esterco, 'Caipira', nutrição, Gigaspora margarita, banana.

\section{SUBSTRATES AND THE ARBUSCULAR MYCORRHIZAL FUNGUS Gigaspora margarita IN THE GROWTH OF MICROPOPAGETED BANANAS PLANTS}

\begin{abstract}
With the aim of studying different substrates and the arbuscular mycorrhizal fungus Gigaspora margarita in the growth of micropropagated banana plants it was conducted an experiment under greenhouse conditions at the CAMPO-CPA (micropropagating plant)/EmbrapaBrazilian Agricultural Research Corporation, in the city of Cruz das Almas, State of Bahia. Thirteen substrates were tested with and without inoculation with the arbuscular mycorrhizal fungus, during the acclimatization phase. The plants were inoculated at the time of transplanting to the different substrates and were collected after the acclimatization period of 55 days, for evaluation of its growth, mineral nutrition and mycorrhizal colonization. The arbuscular mycorrhizal fungus G. margarita colonized well and increased the growth of the micropropagated banana plants, depending on the substrate used; the substrate Turfgrass + vermiculite $+5 \%$ manure promoted the formation of normal and healthy plants but only in the presence of the mycorrhizal fungus; the use of the commercial substrate Rendmax Citrus gave the best plant growth but inhibited mycorrhizal colonization and effects; addition of nutrients to Rendmax Citrus was not necessary for banana plantlets; vermicompost was a good substrate for banana plantlet associated with AM inoculation.
\end{abstract}

Index terms: Musa spp., mycorrhizae, manure, 'Caipira', nutrition, Gigaspora margarita, banana.

\section{INTRODUÇÃO}

O aumento do consumo mundial, com a abertura de novos mercados e a maior exigência dos consumidores brasileiros, está indicando um novo rumo a ser tomado pela bananicultura nacional. $\mathrm{Na}$ busca de novas áreas de plantio e na renovação de áreas pouco produtivas, é fundamental a introdução de mudas de bananeira com alta qualidade genética e fitossanitária. A micropropagação é uma técnica de cultura de tecidos muito importante para a multiplicação massal da bananeira, proporcionando uma taxa superior ao método convencional e obtenção de material livre de doenças e pragas (Souza et al., 1994). Entretanto, as técnicas de micropropagação privam as plântulas de sua microbiota natural benéfica, como os fungos micorrízicos arbusculares (FMAs), embora promovam condições ambientais ótimas em relação à água, nutrição e luz. A condição axênica é geralmente estendida para os estágios iniciais da aclimatação, quando as plantas são transferidas para solos ou substratos.

A micorriza constitui-se numa simbiose praticamente universal, entre os FMAs e as plantas, tanto pelo grande número de plantas suscetíveis à micorrização, como por sua ocorrência generalizada na maioria dos habitats. Várias fruteiras, entre as quais a bananeira, respondem à inoculação dos FMAs em fase de aclimatação. Declerck et al. (1995) demonstraram variação de dependência micorrízica da bananeira (Musa acuminata, grupo AAA). Esta dependência é acentuada principalmente quando a bananeira é oriunda de mudas micropropagadas (Rizzardi, 1990; Monteiro et al. 1991; Declerck et al., 1994 e 1995). Geralmente, o melhor momento para inoculação é no início da fase pós-vitro, embora isto varie com a espécie de planta a ser produzida. Jaizme-Vega et al. (1997), ao inocularem plântulas micropropagadas de bananeira Grand Nine com o fungo G. mosseae, observaram que os efeitos de crescimento apareceram nos primeiros estágios de desenvolvimento pós-vitro e tornaram-se evidentes durante a fase de endurecimento. A multiplicidade de condições utilizadas na obtenção de mudas micropropagadas dificulta a utilização racional dos fungos micorrízicos, sendo necessário estudar as condições ideais para a maximização do efeito da associação. As condições testadas são muito variadas em termos de tipo e fertilidade de substrato, intensidade luminosa, umidade e aeração, entre outros fatores, sendo que todos estes citados são reconhecidamente moduladores do efeito da inoculação sobre as plantas.

Substratos hortícolas para a produção de mudas vêm cada vez mais substituindo o uso do solo mineral como meio de cultivo, propiciando significativos aumentos na produção. Especialmente nos cultivos em recipientes, a qualidade do substrato torna-se mais importante, pois as relações entre o sistema radicular e o substrato são alteradas. A inoculação de FMAs em larga escala para produção de mudas micorrizadas requer que as condições essenciais ao estabelecimento e adequado desempenho da micorriza sejam assegurados. Neste caso, o nível de fertilidade do substrato é um dos principais fatores que devem ser devidamente controlados. Silveira et al. (1995), tentando determinar a dose de matéria orgânica adequada ao desenvolvimento de mudas micorrizadas de mangueira, constataram que as plantas não micorrizadas tiveram uma resposta linear às doses de esterco de curral aplicadas, enquanto as micorrizadas apresentaram resposta quadrática, com um pico de produção de matéria seca da parte aérea e altura na dose de $10 \%$ de esterco. Trindade et al. (2000b)

\footnotetext{
${ }^{1}$ (Trabalho 072/2002). Recebido: 02/04/2002. Aceito para publicação: 09/04/2003.

${ }^{2}$ Embrapa Mandioca e Fruticultura, Cruz das Almas-BA, C. P. 007, CEP: 44380-000; aldo@cnpmf.embrapa.br.

${ }^{3}$ Secretaria de Produção e Reforma Agrária do Estado de Pernambuco, Recife, 50741-380.

${ }^{4}$ Agrônoma, mestranda da Capes, CPG em Solos da AGRUFBA, Cruz das Almas-BA, 44380-000.
} 
verificaram que doses de esterco acima de $10 \%$ reduziram a colonização micorrízica em mudas de mamoeiro. Para banana, não há relatos sobre estes efeitos e para mudas micropopagadas, tem-se usado substratos comerciais, à base de turfa, cascas, esterco e complementação mineral.

Este trabalho objetivou avaliar diferentes substratos na formação de mudas de bananeira 'Caipira' e seu efeito na resposta da planta à inoculação do FMA Gigaspora margarita.

\section{MATERIALEMÉTODOS}

Foi conduzido um experimento em estufa de aclimatação na Embrapa Mandioca e Fruticultura, em Cruz das Almas-BA, com início em fevereiro de 1999. Utilizaram-se mudas micropropagadas de bananeira, variedade Caipira, produzidas pela Biofábrica CAMPO CPA / Embrapa Mandioca e Fruticultura. As mudas foram obtidas por meio de cultura de meristema a partir de plantas do pomar da Embrapa, de acordo com o protocolo utilizado na rotina de produção. As plântulas utilizadas encontravam-se no segundo subcultivo, tendo sido multiplicadas em meio MS (Murashige \& Skoog, 1962) suplementado com $3 \mathrm{mg} / \mathrm{l}$ de 6-benzilaminopurine, $30 \mathrm{~g} / \mathrm{l}$ de sacarose, $2 \mathrm{~g} / \mathrm{l}$ de phytagel. Para enraizamento, utilizou-se o meio MS com 0,25 $\mathrm{mg} / \mathrm{l}$ de ácido naftaleno acético, $30 \mathrm{~g} / \mathrm{l}$ de sacarose e $6 \mathrm{~g} / \mathrm{l}$ de ágar. As plântulas apresentavam, em média, altura de $10,3 \mathrm{~cm}, 5$ folhas, 10 raízes e a maior raiz com $8,9 \mathrm{~cm}$ de comprimento.

Foram testados treze substratos, dois tratamentos fúngicos (inoculado e não inoculado), com cinco repetições, num delineamento experimental de blocos casualizados. Cada parcela foi constituída por um vaso plástico com capacidade de $1,3 \mathrm{dm}^{3}$ contendo uma muda. Os substratos foram compostos pela mistura de componentes orgânicos, previamente secos à sombra, e minerais, com base em volume, conforme descritos na Tabela 1, e incubados nos vasos, com água destilada, por 15 dias. Após esse período, amostras foram retiradas para análises químicas e físicas (Tabela 2). Visando à eliminação de qualquer propágulo de FMAs, todos os componentes que formaram as misturas e também os substratos comerciais, foram fumigados com brometo de metila $\left(393 \mathrm{ml} / \mathrm{m}^{3}\right.$, aplicados ao substrato contido em caixa de cimento amianto e com tempo de retenção de 48 horas). Posteriormente, para o Rendmax citrus e o Rendmax crisântemo, ambos sem complementação mineral, realizou-se a correção do $\mathrm{pH}$ com $\mathrm{CaCO}_{3}$ P.A., com uma dosagem equivalente a 1,5 t/ha. Para o enchimento dos vasos, foi utilizado $1,2 \mathrm{dm}^{3}$ de cada substrato, sendo posteriormente umedecidos com água destilada, permanecendo em incubação por 15 dias. Decorrido este período, as mudas provenientes da fase in vitro foram transplantadas e inoculadas com o fungo micorrízico Gigaspora margarita, utilizando-se de $25 \mathrm{~g}$ do inóculo, o qual continha aproximadamente 416 esporos. $\mathrm{O}$ inóculo foi colocado abaixo da muda, para que as raízes ficassem em contato com o mesmo. Este inóculo, composto por esporos, solo e raízes colonizadas, foi obtido da coleção da Embrapa Mandioca e Fruticultura, tendo sido multiplicado anteriormente em plantas de sorgo cultivadas por quatro meses em mistura de solo:areia $(2: 1 \mathrm{v}: \mathrm{v})$. Os tratamentos não inoculados receberam $10 \mathrm{ml}$ de um filtrado do inóculo, obtido após passagem por peneira de 400 mesh de abertura, visando a recompor a microbiota do inóculo, fora os propágulos do fungo micorrízico.

Aos 55 dias após o transplantio, as mudas foram coletadas,

TABELA 1- Composição dos substratos utilizados no experimento

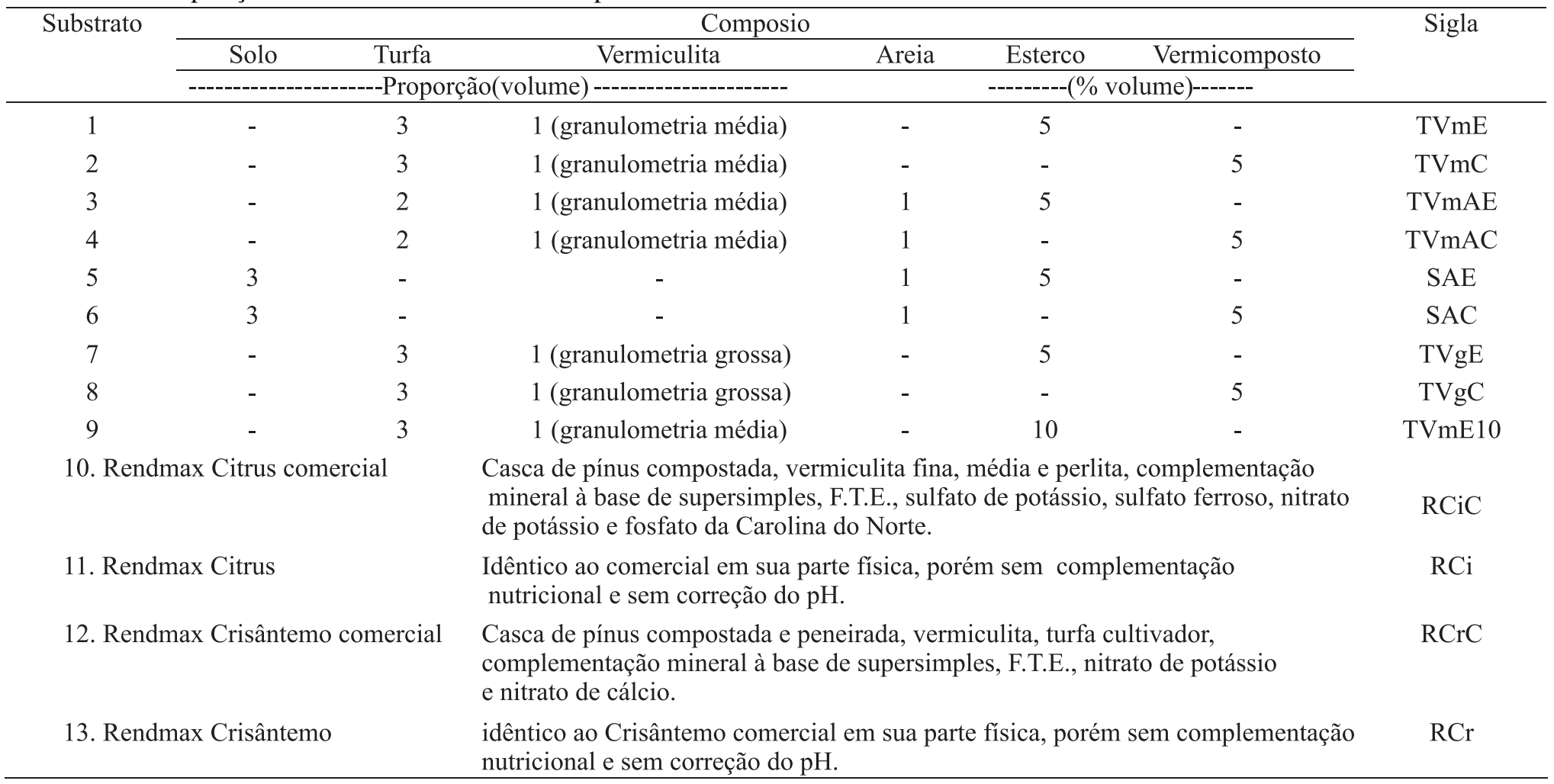

separando-se a parte aérea do sistema radicular na região do colo. Da parte aérea, obteve-se o peso de matéria fresca e matéria seca. A matéria seca foi obtida pesando-se a parte aérea, após secagem por 72 horas a $65^{\circ} \mathrm{C}$. Esta foi moída e uma amostra submetida à digestão nítrico-perclórica para análise de $\mathrm{P}$ por fotometria de luz vizível, $\mathrm{Cu}$ e $\mathrm{Zn}$ por expectrofotometria de absorção atômica. O sistema radicular foi separado em raízes grossas (raízes - cordiformes, brancas e tenras) e finas (radicelas - escuras, em forma de cabeleira), obtendo-se o comprimento, respectivamente, pela medição com auxílio de régua e pelo método da placa reticulada (Newman, 1966). As radicelas foram utilizadas posteriormente para coloração com azul de tripan (Phillips \& Hayman, 1970) e determinação da taxa de colonização pelo método da interseção linear (Ambler \& Young, 1977).

Os dados obtidos foram analisados pelo programa SAEG, sendo efetuados análise de variância (Teste F) e teste de média (Tukey) para comparar tratamentos de inoculação, substrato e a interação. 
TABELA 2- Características químicas e físicas dos substratos.

\begin{tabular}{|c|c|c|c|c|c|c|c|c|c|c|c|c|c|}
\hline \multirow[t]{2}{*}{ Substrato } & \multirow[t]{2}{*}{$\mathrm{pH}$} & \multirow{2}{*}{$\begin{array}{l}\mathrm{P} \\
\mathrm{H}_{2} \mathrm{O} \\
\end{array}$} & \multirow{2}{*}{\multicolumn{2}{|c|}{$\begin{array}{l}\mathrm{K} \quad \mathrm{Ca} \\
--\mathrm{mg} / \mathrm{dm}^{3}--\end{array}$}} & \multirow{2}{*}{$\mathrm{Mg}$} & $\mathrm{A} 1$ & $\mathrm{Na}$ & \multirow{2}{*}{$\begin{array}{l}\text { CTC } \\
----- \\
\end{array}$} & \multirow{2}{*}{$\begin{array}{l}\text { M.O. } \\
\text { g/dm }\end{array}$} & \multirow{2}{*}{$\begin{array}{c}\text { C.E. } \\
\mathrm{mmho} / \mathrm{cm}\end{array}$} & \multicolumn{2}{|c|}{ Densidade } & \multirow{2}{*}{$\begin{array}{r}\text { Retenção } \\
\text { de água }\end{array}$} \\
\hline & & & & & & $---\mathrm{cl}$ & $\mathrm{d} / \mathrm{dm}^{3}$ & & & & Partícula & Aparente & \\
\hline & & & & & & & & & & & --------g/c & $m^{3}-------$ & $(0,33 \mathrm{~atm})$ \\
\hline TVmE & 5,47 & 38 & 400 & 3,6 & 2,9 & 0,8 & 0,55 & 29,75 & 122,9 & 1,49 & 2,12 & 1,00 & 31,86 \\
\hline TVmC & 5,03 & 32 & 140 & 4,6 & 3,1 & 0,7 & 0,26 & 31,64 & 129,8 & 1,72 & 2,00 & 1,07 & 25,48 \\
\hline TVmAE & 5,65 & 48 & 350 & 3,2 & 2,6 & 0,4 & 0,45 & 22,77 & 72,4 & 1,99 & 2,18 & 1,22 & 15,58 \\
\hline TVmAC & 5,62 & 50 & 150 & 5,1 & 3,8 & 0,2 & 0,24 & 24,04 & 69,0 & 2,10 & 2,21 & 1,05 & 18,72 \\
\hline SAE & 7,65 & 48 & 190 & 3,3 & 1,1 & 0,0 & 0,21 & 5,10 & 15,0 & 1,80 & 2,60 & 1,55 & 4,63 \\
\hline SAC & 7,50 & 46 & 83 & 3,8 & 1,2 & 0,0 & 0,13 & 5,34 & 14,3 & 2,22 & 2,16 & 1,07 & 25,30 \\
\hline TVgE & 5,43 & 39 & 540 & 3,2 & 2,3 & 0,4 & 0,70 & 28,81 & 128,1 & 1,99 & 2,11 & 1,10 & 25,56 \\
\hline $\mathrm{TVgC}$ & 5,02 & 28 & 150 & 4,4 & 2,6 & 0,6 & 0,27 & 29,21 & 140,0 & 1,34 & 1,91 & 1,02 & 30,62 \\
\hline TVmE10 & 5,40 & 64 & 1040 & 4,3 & 3,8 & 0,1 & 1,30 & 30,39 & 104,2 & 2,30 & 1,75 & 0,93 & 29,45 \\
\hline $\mathrm{RCiC}$ & 4,32 & 115 & 380 & 4,8 & 3,2 & 0,6 & 0,31 & 26,77 & 314,2 & 2,40 & 1,06 & 0,38 & 88,84 \\
\hline $\mathrm{RCi}$ & 4,95 & 36 & 140 & 6,0 & 4,0 & 0,3 & 0,40 & 23,19 & 321,0 & 1,35 & 1,06 & 0,38 & 88,84 \\
\hline $\mathrm{RCrC}$ & 5,75 & 93 & 160 & 8,6 & 5,2 & 0,0 & 0,32 & 22,34 & 304,0 & 1,97 & 1,09 & 0,40 & 73,41 \\
\hline $\mathrm{RCr}$ & 5,35 & 60 & 160 & 6,2 & 5,9 & 0,1 & 0,38 & 20,59 & 228,8 & 0,78 & 1,09 & 0,40 & 73,41 \\
\hline
\end{tabular}

\section{RESULTADOSE DISCUSSÃO}

O uso de diferentes substratos promoveu efeito significativo para todas as características avaliadas. O efeito da inoculação ou da sua interação com os substratos foi significativo com relação à colonização, teores de $\mathrm{P}$ e Zn e características de crescimento, com exceção do comprimento de radicela.

Para o peso de parte aérea fresca, os substratos Rendmax Citrus, com (RCiC) ou sem complementação mineral ( $\mathrm{RCi})$, destacaram-se como os melhores (Tabela 3). O substrato TVmE também se destacou, não diferindo dos RCiC e RCi, para plantas inoculadas. Além do TVmE, a inoculação favoreceu a produção de matéria fresca das plantas nos substratos TVmC, TVmAC e TVgC, todos apresentando turfa e vermiculita em sua composição. O fungo G. margarita não está entre as espécies mais testadas para a bananeira, mas tem promovido grandes respostas no crescimento de fruteiras, como macieira (Kon, 1995), goiabeira (Samarão \& Martins, 1998) e mamoeiro (Trindade et al., 2000a). O uso de areia, em substituição à parte da turfa, eliminou o efeito benéfico do fungo no crescimento das mudas de banana, quando do uso de esterco na mistura de cultivo. Já a substituição total da turfa por solo e areia não permitiu o efeito do fungo, tanto na presença de esterco como na de vermicomposto. A substituição de vermiculita de granulometria média por uma de granulometria maior manteve o efeito benéfico da inoculação no crescimento das plantas quando do uso de vermicomposto em mistura, não se verificando o mesmo para o esterco. $\mathrm{O}$ aumento da dose de esterco de 5\% para 10\% não trouxe benefícios para o cultivo das mudas de bananeiras, possivelmente por um aumento na condutividade elétrica, já que os teores de potássio atingiram valores acima de $1000 \mathrm{mg} /$ $\mathrm{dm}^{3}$. Também não foi favorável à eficiência micorrízica, o que pode ser atribuído ao aumento dos teores de $\mathrm{P}$ do substrato (Tabela 2). A eficiência da associação micorrízica em banana apresentou-se limitada pela presença do esterco, já que resultados com outras fruteiras mostram que este resíduo pode beneficiar a simbiose em doses de $10 \%$, para o mamoeiro (Trindade et al., 2000b) e 40\%, para o maracujazeiro (Silveira et al., 1996). Deve-se considerar, entretanto, que a composição do esterco nem sempre é constante, variando, entre outros fatores, com o grau de confinamento adotado para o rebanho bovino que origina o resíduo.

TABELA 3- Produção de parte aérea fresca (MFPA) e seca (MSPA) em função de substratos de crescimento e da inoculação com G. margarita.

\begin{tabular}{|c|c|c|c|c|c|c|c|}
\hline \multirow{2}{*}{ Substrato } & \multicolumn{4}{|c|}{ MFPA (g/planta) * } & \multicolumn{3}{|c|}{ MSPA (g/planta) * } \\
\hline & \multicolumn{2}{|c|}{ Inoculado } & \multicolumn{2}{|c|}{ Não inoculado } & Inoculado & Não inoculado & Média \\
\hline TVmE & 32,02 & $\mathrm{ABCa}$ & 21,00 & $\mathrm{BCb}$ & 2,61 & 1,74 & $2,17 \mathrm{BC}$ \\
\hline TVmC & 26,04 & $\mathrm{BCDa}$ & 13,16 & $\mathrm{BCDb}$ & 2,22 & 1,12 & $1,67 \mathrm{CD}$ \\
\hline TVmAE & 20,00 & $\mathrm{CDa}$ & 19,36 & $\mathrm{BCDa}$ & 1,52 & 1,60 & $1,56 \mathrm{CD}$ \\
\hline TVmAC & 26,58 & $\mathrm{BCDa}$ & 17,05 & $\mathrm{BCDb}$ & 2,17 & 1,65 & $1,91 \mathrm{CD}$ \\
\hline SAE & 15,90 & $\mathrm{DEa}$ & 9,82 & $\mathrm{CDa}$ & 1,39 & 0,78 & $1,09 \mathrm{DE}$ \\
\hline SAC & 20,14 & $\mathrm{CDa}$ & 19,48 & $\mathrm{BCDa}$ & 1,93 & 1,74 & $1,83 \mathrm{CD}$ \\
\hline TVgE & 23,60 & $\mathrm{CDa}$ & 21,38 & $\mathrm{BCa}$ & 1,76 & 1,85 & $1,80 \mathrm{CD}$ \\
\hline TVgC & 26,56 & $\mathrm{BCDa}$ & 16,70 & $\mathrm{BCDb}$ & 2,22 & 1,58 & $1,90 \mathrm{CD}$ \\
\hline TVmE10 & 23,10 & $\mathrm{CDa}$ & 23,54 & $\mathrm{Ba}$ & 1,68 & 1,80 & $1,74 \mathrm{CD}$ \\
\hline $\mathrm{RCiC}$ & 37,84 & $\mathrm{ABa}$ & 44,32 & $\mathrm{Aa}$ & 2,84 & 3,23 & $3,03 \mathrm{AB}$ \\
\hline $\mathrm{RCi}$ & 40,20 & $\mathrm{Aa}$ & 37,02 & $\mathrm{Aa}$ & 3,63 & 3,13 & $3,38 \mathrm{~A}$ \\
\hline $\mathrm{RCrC}$ & 21,98 & $\mathrm{CDa}$ & 21,76 & $\mathrm{BCa}$ & 1,90 & 1,90 & $1,90 \mathrm{CD}$ \\
\hline $\mathrm{RCr}$ & 5,26 & $\mathrm{Ea}$ & 7,36 & $\mathrm{Da}$ & 0,45 & 0,54 & $0,49 \mathrm{E}$ \\
\hline Média & & & & & $2,02 a$ & $1,74 b$ & \\
\hline
\end{tabular}

* Médias seguidas pela mesma letra não diferem significativamente, pelo teste de Tukey, ao nível de 5\% de probabilidade. As letras maiúsculas nas colunas e minúsculas nas linhas representam as comparações entre substratos e inoculação respectivamente.

Para os substratos comerciais, a inoculação não trouxe benefícios para a produção de parte aérea das mudas de bananeira. Entretanto, a eliminação da complementação mineral não modificou significativamente o efeito dos fungos. Para a maioria dos substratos testados, particularmente aqueles com elevados teores de matéria orgânica, é provável que o extrator químico usado na determinação do fósforo, Mehlich-1, não tenha estimado com rigor os teores disponíveis por ser mais apropriado para solos com $\mathrm{pH}$ ácido e baixos teores de matéria orgânica. Por este método empregado, os substratos comerciais apresentaram teores de $\mathrm{P}$ semelhantes ou inferiores a ou- 
tros em que a inoculação foi eficiente. Segundo informações do fabricante, no processo de compostagem para a obtenção do composto organomineral, é feita uma adição de nutrientes para tornar o meio mais propício à atuação dos microrganismos decompositores.

Para a matéria seca da parte aérea, apenas os efeitos principais (Substrato e Inoculação) foram significativos. Entre os substratos, destacaram-se os comerciais RCiC e RCi, seguidos da mistura TVmE. $\mathrm{O}$ uso de vermicomposto, misturado com qualquer dos demais componentes dos substratos, não diferiu significativamente dos resultados obtidos com o uso de esterco. Comparando-se os substratos TVmE e SAE, verifica-se que, em combinação com o esterco, a melhor mistura foi turfa + vermiculita. $\mathrm{O}$ uso de solo e areia não foi benéfico para a produção de matéria seca da planta, principalmente com o uso de esterco em mistura. A substituição de turfa por areia e vermiculita média por uma de granulometria grossa não promoveu alterações significativas no peso de matéria seca da parte aérea das plantas.

O Rendmax Crisântemo limitou o desenvolvimento das plantas. A diferença básica deste substrato para o Rendmax Citrus está no acréscimo de turfa. Embora o uso desse componente seja disseminado e normalmente conduza a uma boa condição de cultivo, existem diferentes tipos de turfa, em função do material originário e condições edafoclimáticas de sua formação. Caron \& Parent (1988) afirma- ram que a eficiência de FMAs, bem como o crescimento da planta, pode ser alterada pela natureza da turfa utilizada.

O comprimento de raiz foi influenciado pela interação entre Inoculação e Substratos (Tabela 4). Entretanto, diferentemente da produção de parte aérea, a inoculação promoveu alterações significativas, naquela variável, apenas na presença dos substratos TVmC e SAC, ambos contendo vermicomposto. Entre as plantas inoculadas, os substratos tiveram pouca influência. Para as plantas não inoculadas, SAE e RCr foram os piores substratos; o uso de esterco, em relação ao vermicomposto, não provocou diferenças significativas em nenhuma combinação com outros componentes e a dose de $10 \%$, assim como foi observado para a parte aérea, não alterou a produção de raiz. O vermicomposto poderia substituir o esterco na maioria das misturas empregadas, sem comprometer o crescimento da parte aérea ou raiz. O efeito positivo da inoculação sobre o crescimento da planta foi mais regular com o uso de vermicomposto, embora não tão intenso como o verificado na mistura TVmE. O vermicomposto tem sido recomendado para a produção de mudas de espécies arbóreas (Vieira et al., 1998; Tedesco et al., 1999) em doses de até 30\% . O uso de Rendmax Citrus sem complementação mineral promoveu os maiores comprimentos de raiz, sendo superiores à maior parte dos substratos contendo vermicomposto, aos substratos contendo solo

TABELA 4- Comprimento de raízes e radicelas de mudas de bananeira em função do substrato de crescimento e da inoculação com G. margarita.

\begin{tabular}{|c|c|c|c|c|c|c|}
\hline \multirow[b]{2}{*}{ Substrato } & \multicolumn{3}{|c|}{ Raiz (cm/planta)* } & \multicolumn{3}{|c|}{ Radicela (cm/planta)* } \\
\hline & Inoculado & Não ir & culado & Inoculado & Não inoculado & Média \\
\hline TvmE & $348 \mathrm{ABa}$ & 343 & $\mathrm{ABCa}$ & 2446 & 3891 & $3168 \mathrm{~B}$ \\
\hline TVmC & $320 \mathrm{ABa}$ & 196 & $\mathrm{CDb}$ & 4129 & 2118 & $3123 \mathrm{~B}$ \\
\hline TVmAE & $232 \mathrm{ABa}$ & 352 & $\mathrm{ABCa}$ & 2097 & 2712 & $2405 \mathrm{BC}$ \\
\hline $\mathrm{TVmAC}$ & $246 \mathrm{ABa}$ & 211 & $\mathrm{CDa}$ & 2674 & 3096 & $2885 \mathrm{~B}$ \\
\hline SAE & $242 \mathrm{ABa}$ & 141 & $\mathrm{Da}$ & 1012 & 923 & $967 \mathrm{CDE}$ \\
\hline SAC & $318 \mathrm{ABa}$ & 192 & $\mathrm{CDb}$ & 1279 & 690 & $985 \mathrm{DE}$ \\
\hline TVgE & $308 \mathrm{ABa}$ & 368 & $\mathrm{ABCa}$ & 1484 & 4132 & $2808 \mathrm{BCDE}$ \\
\hline TVgC & $372 \mathrm{Aa}$ & 298 & $\mathrm{ABCDa}$ & 3578 & 2197 & $2887 \mathrm{BC}$ \\
\hline TVmE10 & $222 \mathrm{ABa}$ & 250 & $\mathrm{BCDa}$ & 1415 & 3093 & 2254 BCD \\
\hline $\mathrm{RCiC}$ & $397 \mathrm{Aa}$ & 474 & $\mathrm{Aa}$ & 2858 & 2601 & $2730 \mathrm{BC}$ \\
\hline $\mathrm{RCi}$ & $354 \mathrm{ABa}$ & 416 & $\mathrm{ABa}$ & 9521 & 9474 & $9497 \mathrm{~A}$ \\
\hline $\mathrm{RCrC}$ & $295 \mathrm{ABa}$ & 326 & $\mathrm{ABCDa}$ & 1869 & 1909 & $1889 \mathrm{BCD}$ \\
\hline $\mathrm{RCr}$ & $165 \mathrm{Ba}$ & 210 & $\mathrm{CDa}$ & 554 & 651 & $602 \mathrm{E}$ \\
\hline
\end{tabular}

* Médias seguidas pela mesma letra não diferem significativamente, pelo teste de Tukey, ao nível de $5 \%$ de probabilidade. Letras maiúsculas para comparação dos substratos e letras minúsculas para comparação da inoculação.

Para efeito da análise estatística, os dados foram transformados para [log $(\mathrm{x}+1)]$.

+ areia e ao Rendmax Crisântemo sem complementação mineral.

Apesar da grande amplitude de valores entre plantas submetidas ou não à inoculação, o comprimento de radicelas sofreu influência apenas dos substratos (Tabela 4). Verifica-se que os substratos TVmE e RCiC que favoreceram o crescimento de parte aérea e de raiz, tiveram efeito reduzido na produção de radicelas. Semelhantemente ao que ocorreu para raiz, os substratos com uso de solo + areia e Rendmax Crisântemo sem complementação mineral comprometeram a produção de radicelas. A ausência de complementação mineral no substrato Rendmax Citrus resultou em aumento altamente significativo na produção de radicelas, sendo maior que todos os demais substratos, embora não tenha alterado o acúmulo de biomassa na parte aérea (Tabela 4). Isto sugere um efeito direto do substrato sobre o crescimento das raízes, que pode ter sido a razão do desenvolvimento de parte aérea equivalente àquele verificado no mesmo substrato com complementação mineral. A ausência de complemento mineral, entretanto, promoveu efeitos inversos no substrato Rendmax Crisântemo, sugerindo que este substrato não apresenta um bom equilíbrio de nutrientes para o cultivo da bananeira, como ficou comprovado pelo desenvolvimento de parte aérea e maior formação pro- porcional de raízes grossas, com baixa capacidade de absorção de água e nutrientes. Este é um dado importante quando se pensa no estabelecimento da muda em campo após o transplantio, pois aquelas plantas com maior aparato de absorção, seja na forma de radicelas seja na de micorrizas, deverão ter melhor desenvolvimento inicial. A substituição de parte da turfa por areia como forma de aumentar a aeração e, conseqüentemente, favorecer a planta, não se mostrou efetiva além de eliminar o efeito benéfico dos fungos quando do uso de esterco na mistura, o que pode estar associado à menor produção de radicelas pelas plantas.

A taxa de colonização de mudas de bananeira inoculadas com G. margarita variou de $5 \%$ a $90 \%$ nos diferentes substratos (Figura 1). O efeito da inoculação no substrato TVmE e nos demais em que também houve significância, apresenta respaldo nos valores elevados de percentagem de colonização radicular. Todos estes substratos apresentavam mistura de turfa e vermiculita. Este tipo de mistura com ou sem adição de esterco foi recomendada por Zambolim et al. (1992) como substrato para produção de inóculo de fungos micorrízicos arbusculares por promover elevados percentuais de 


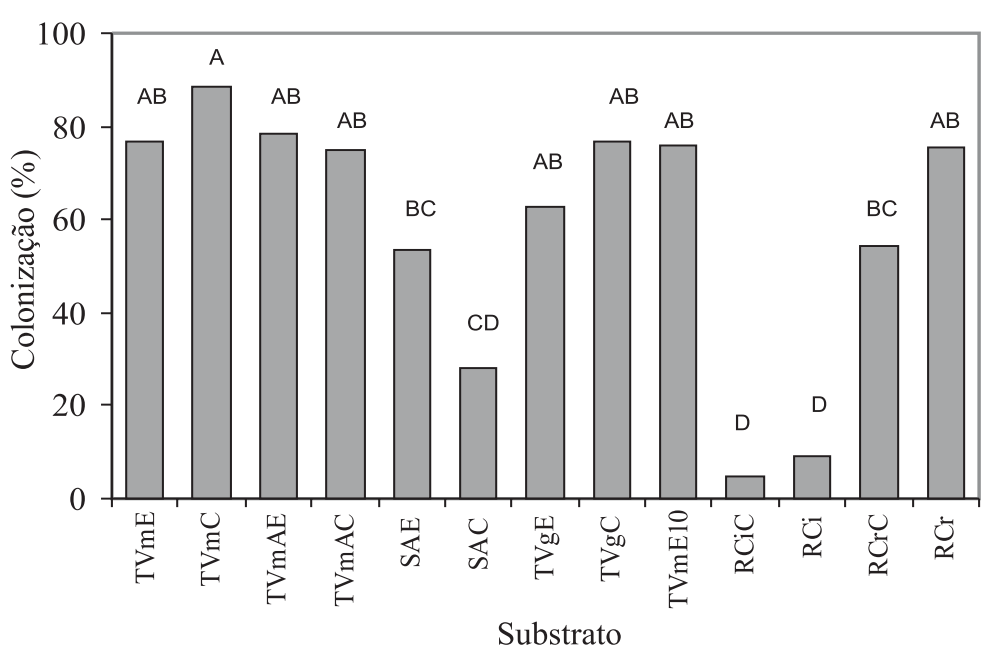

FIGURA 1-Percentagem de colonização micorrízica de mudas de bananeira por G. margarita em função do substrato de cultivo. Médias seguidas pela mesma letra não diferem significativamente, pelo teste de Tukey, ao nível de 5\% de probabilidade.

colonização e produção de esporos.

Com o aumento da dose de esterco de 5\% (TVmE) para 10\% (TVmE10), a colonização manteve-se alta (Figura 1), porém sem eficiência para o crescimento da planta. $\mathrm{O}$ aumento de componentes minerais, no caso solo e areia, em substituição à turfa e vermiculita, foi prejudicial para a colonização micorrízica, assim como aconteceu para o acúmulo de parte aérea e produção de radicelas. Pode-se atribuir o efeito da mistura solo e areia à sua elevada densidade aparente e baixa retenção de umidade (particularmente no caso de SAE), mas, de todos os substratos, foram os únicos com $\mathrm{pH}$ na faixa alcalina, devido provavelmente ao menor poder tampão da areia, o que pode ter sido decisivo para o seu efeito negativo. O substrato Rendmax Citrus, mesmo quando não recebeu complementação mineral, também promoveu baixas taxas de colonização.

As plantas que apresentaram maiores teores de $\mathrm{P}$ foram as que se desenvolveram nos substratos Rendmax (Tabela 5). O uso de vermicomposto em substituição ao esterco não provocou alterações significativas na absorção de $\mathrm{P}$. O mesmo se verificou quando da substituição de vermiculita por areia ou quando do uso de vermiculita grossa no lugar de vermiculita média. $\mathrm{O}$ aumento da dose de esterco não alterou significativamente a concentração de $\mathrm{P}$, mas permitiu maior teor nas plantas inoculadas. Além do TVmE10, a inoculação aumentou os teores de $\mathrm{P}$ das plantas cultivadas também nos substratos TVmAE, TVmAC e TVgE. Verifica-se que, embora os valores de colonização tenham sido altos para muitos dos tratamentos testados, nem sempre se refletiram no crescimento da parte aérea, mas resultaram em maiores teores de fósforo na planta, como no caso dos substratos TVmAE, TVgE e TVmE10. Embora o fósforo não esteja entre os macronutrientes mais absorvidos pela bananeira (Borges \& Silva, 1995), os trabalhos feitos com a inoculação de FMAs nesta cultura têm mostrado grandes respostas na absorção de fósforo

TABELA 5- Teores de P, Zn e Cu na parte aérea das mudas de bananeira em função do substrato de crescimento e da inoculação com G. margarita.

\begin{tabular}{|c|c|c|c|c|c|c|c|c|c|c|c|c|}
\hline \multirow{3}{*}{$\frac{\text { Substrato }}{\text { TVmE }}$} & \multicolumn{4}{|c|}{$\mathrm{P}(\mathrm{g} / \mathrm{kg})$} & \multicolumn{4}{|c|}{$\mathrm{Zn}(\mathrm{mg} / \mathrm{g})$} & \multicolumn{4}{|c|}{$\mathrm{Cu}(\mathrm{mg} / \mathrm{g})$} \\
\hline & \multicolumn{2}{|c|}{ Inoculado } & \multicolumn{2}{|c|}{ Não inoculado } & \multicolumn{2}{|c|}{ Inoculado } & \multicolumn{2}{|c|}{ Não inoculado } & \multirow{2}{*}{$\begin{array}{l}\text { Inoculado } \\
11,40\end{array}$} & \multirow{2}{*}{$\begin{array}{c}\text { Não inoculado } \\
10,20\end{array}$} & \multicolumn{2}{|l|}{ Média } \\
\hline & 1,02 & $\mathrm{Ea}$ & 0,94 & $\overline{B C D a}$ & 49,60 & $\mathrm{BCDEa}$ & 53,20 & $\overline{\mathrm{ABa}}$ & & & 10,80 & B \\
\hline TVmC & 0,92 & $\mathrm{Ea}$ & 0,76 & $\mathrm{CDa}$ & 82,80 & $\mathrm{ABCa}$ & 71,80 & $\mathrm{Aa}$ & 14,60 & 11,40 & 13,00 & B \\
\hline TVmAE & 1,52 & $\mathrm{DEa}$ & 0,64 & $\mathrm{Db}$ & 65,20 & $\mathrm{ABCDa}$ & 28,80 & $\mathrm{Bb}$ & 9,40 & 9,20 & 9,30 & B \\
\hline TVmAC & 1,40 & $\mathrm{DEa}$ & 0,78 & $\mathrm{CDb}$ & 85,40 & $\mathrm{ABa}$ & 58,00 & $\mathrm{ABb}$ & 16,40 & 10,60 & 13,50 & B \\
\hline SAE & 2,14 & $\mathrm{BCDa}$ & 1,86 & $\mathrm{Ba}$ & 25,80 & $\mathrm{Ea}$ & 47,80 & $\mathrm{ABa}$ & 22,60 & 27,80 & 25,20 & A \\
\hline SAC & 1,66 & $\mathrm{CDEa}$ & 1,62 & $\mathrm{BCa}$ & 47,60 & $\mathrm{BCDEa}$ & 48,80 & $\mathrm{ABa}$ & 19,80 & 22,00 & 20,90 & A \\
\hline TVgE & 1,30 & $\mathrm{DEa}$ & 0,68 & $\mathrm{Db}$ & 51,20 & ABCDEa & 47,80 & $\mathrm{ABa}$ & 11,80 & 12,60 & 12,20 & B \\
\hline TVgC & 1,12 & $\mathrm{Ea}$ & 0,78 & $\mathrm{CDa}$ & 89,20 & $\mathrm{Aa}$ & 56,00 & $\mathrm{ABb}$ & 16,60 & 10,80 & 13,70 & B \\
\hline TVmE10 & 1,82 & $\mathrm{BCDEa}$ & 0,86 & $\mathrm{CDb}$ & 51,80 & ABCDEa & 49,60 & $\mathrm{ABa}$ & 11,00 & 11,80 & 11,40 & B \\
\hline $\mathrm{RCiC}$ & 2,62 & $\mathrm{ABa}$ & 3,14 & $\mathrm{Aa}$ & 44,20 & $\mathrm{CDEa}$ & 50,00 & $\mathrm{ABa}$ & 15,20 & 13,40 & 14,30 & B \\
\hline $\mathrm{RCi}$ & 2,54 & $\mathrm{ABCb}$ & 3,14 & $\mathrm{Aa}$ & 45,20 & $\mathrm{CDEa}$ & 47,60 & $\mathrm{ABa}$ & 12,20 & 14,00 & 13,10 & B \\
\hline $\mathrm{RCrC}$ & 3,08 & $\mathrm{Aa}$ & 3,16 & Aa & 64,60 & $\mathrm{ABCDa}$ & 65,80 & $\mathrm{ABa}$ & 20,60 & 21,20 & 20,90 & A \\
\hline $\mathrm{RCr}$ & 3,46 & $\mathrm{Aa}$ & 2,96 & $\mathrm{Aa}$ & 40,40 & $\mathrm{DEa}$ & 34,60 & $\mathrm{ABa}$ & 15,20 & 13,00 & 14,10 & B \\
\hline
\end{tabular}

Médias seguidas pela mesma letra não diferem significativamente, pelo teste de Tukey, ao nível de 5\% de probabilidade. As letras maiúsculas representam as comparações entre substratos e as minúsculas indicam as comparações entre inoculação.

(Lin \& Fox, 1987; Monteiro et al., 1991; Declerck et al., 1994 e 1995).

A inoculação aumentou significativamente a concentração de Zn das plantas (Tabela 5) crescidas nos substratos onde se substituiu parte da vermiculita por areia (TVmAE e TVmAC) e naquele onde a vermiculita média foi substituída por vermiculita grossa, na presença de vermicomposto ( $\mathrm{TVgC}$ ). O efeito da inoculação, portanto, mostrou-se na maior absorção de fósforo ou zinco. Na ausência de inoculação, os substratos tiveram pouco efeito na concentração de $\mathrm{Zn}$ das plantas. Destaca-se que, diferentemente do que ocorreu para absorção de P, os substratos Rendmax citrus e Rendmax crisântemo sem complementação mineral promoveram baixa concentração de $\mathrm{Zn}$ na parte aérea das plantas, não diferindo do correspondente com complemento. Na presença de esterco, a substituição de turfa e vermiculita por solo causou redução na absorção do zinco. Por outro lado, a substituição de esterco por vermicomposto teve pouco ou nenhum efeito significativo, assim como a complementação mineral dos substratos Rendmax.

Para o $\mathrm{Cu}$, houve efeito apenas dos substratos (Tabela 5). Apesar disso, sua absorção não foi fator crítico, apresentando teores mais elevados ou mais baixos na planta, independentemente do seu grau de crescimento. Diferentemente do comportamento observado para o zinco, os teores de $\mathrm{Cu}$ foram maiores nas plantas cultivadas nos substratos com adição de solo e areia em substituição à turfa e vermiculita, sendo também superiores à maioria dos outros substratos, provavelmente como resultado dos baixos teores de matéria orgânica, que tem grande afinidade de complexação pelo elemento. Por outro lado, a mesma substituição resultou em decréscimo na absorção de zinco pelas plantas, indicando que, neste tipo de substrato, a possibilidade de desordens nutricionais, por falta ou excesso, são maiores.

\section{CONCLUSÕES}

1) O fungo MA Gigaspora margarita colonizou intensamente a bananeira e mostrou-se benéfico para o crescimento das mudas, sendo este efeito modulado pelo substrato de crescimento.

2) O substrato turfa + vermiculita $+5 \%$ de esterco destacou-se entre os melhores para a formação de mudas de bananeira, quando associado à inoculação de fungos MA.

3) $\mathrm{O}$ uso de esterco no substrato de cultivo deve ser feito em 
pequenas doses, de até $5 \%$.

4) O uso do substrato comercial Rendmax Citrus promoveu o melhor desenvolvimento das mudas, mas inibiu a colonização e o efeito da micorriza.

5) A complementação mineral do Rendmax Citrus não foi necessária para o cultivo de mudas de bananeira.

6) $\mathrm{O}$ uso de vermicomposto mostrou-se promissor para a produção de mudas de bananeira, permitindo o efeito da inoculação com fungos MA.

7) A mistura de solo e areia não foi adequada ao desenvolvimento de mudas de bananeira.

\section{REFERÊNCIASBIBLIOGRÁFICAS}

AMBLER, J. R.; YOUNG, J. L. Techniques for determining root length infected by vesicular-arbuscular mycorrhizae. Soil Science Society of American Journal, Madison, v.4, p.551-556, 1977.

BORGES, A.L.; SILVA, S. de O. Extração de macronutrientes por cultivares de banana. Revista Brasileira de Fruticultura, Cruz das Almas, v.17, n.1, p.57-66, 1995.

CARON, M.; PARENT, S. Definition of a peat-lite medium for the use of vesicular-arbuscular mycorrhizae (VAM) in horticulture. Acta Horticulturae, The Hague, v.221, p.289-294, 1988.

DECLERCK, S.; DEVOS, B.; DELVAUX, B.; PLENCHETTE, C. Growth response of micropropagated banana plants to VAM inoculation. Fruits, Paris, v.49, n.2, p.103-109, mar./abr.1994.

DECLERCK, S.; PLENCHETTE, C.; STRULLU, D. G. Mycorrhizal dependency of banana (Musa acuminata, AAA group) cultivar. Plant and Soil, The Hague, v.176, p.183-187, 1995.

JAIZME-VEGA, M. C. et al. Interactions between the root-knot nematode Meloidogyne incognita and Glomus mosseae in banana. Plant and Soil, The Hague, v.196, p.27-35, 1997.

KON, T. Effects of the inoculation with vesicular-arbuscular mycorrhizal fungi on the growth of apple seedlings. Bulletin of the Aomori Apple Experiment Station, Aomori. v.28, p.53-73, 1995.

LIN, M. L.; FOX. R. L. External and internal P requirements of mycorrhizal and non-mycorrhizal banana plants. Journal of Plant Nutrition, New York, v.10, n.9-16, p.1341-1348, 1987.

MONTEIRO, E.M.S; MATOS, R.M.B; PAULA, M.A. de; GUERRA, J.G.M Micorrizas vesículo-arbusculares em bananeiras: Aclimatação e transplante de mudas micropropagadas. In: REUNIÃO BRASILEIRA SOBRE MICORRIZAS, 4. 1991, Mendes, Programa e Resumos..., Itaguaí: EMBRAPA-CNPBS; UFRRJ, 1991.p. 163.

MURASHIGE, T.; SKOOG, F. A revised medium for rapid growth and bioassays with tobacco tissue cultures. Physiologia Plantarum, Copenhagen, v.15, p.473-497, 1962.

NEWMAN, E. I. A method of estimating the total length of root in a sample. Journal of Applied Ecology, Oxford, v.3, n.2, p.139-145,
1966.

PHILLIPS, J. M.; HAYMAN, D. S. Improved procedures for clearing roots and staining parasitic and vesicular-arbuscular mycorrhizal fungi for rapid assessment of infection. Transactions of British Mycological Society, Cambridge, v.55, n.158-161, 1970.

RIZZARDI, V. Effect of inoculation with vesicular-arbuscular mycorrhizal fungi on the growth of micropropagated Musa acuminata clone “Grand Nain”. Rivista di Agricoltura Subtropicale e Tropicale. Firenze, v.84, n.3, p.473-484, 1990.

SAMARÃO, S. S., MARTINS, M. A. Influência da inoculação com fungos micorrízicos arbusculares associada à aplicação de rutina na produção de mudas de goiabeira (Psidium guajava L.). In: FERTBIO, Caxambu, 1998. Resumos... Lavras, Universidade Federal de Lavras, 1998, p. 212.

SILVEIRA, A. P. D.; AZEVEDO, I. C.; OLIVEIRA, E. Obtenção de mudas de mangueira influenciadas pela adição de fungos micorrízicos arbusculares e de matéria orgânica ao substrato. In: CONGRESSO BRASILEIRO DE CIÊNCIADO SOLO, 25., 1995, Viçosa-MG. Resumos... Viçosa, MG: SBCS, 1995. p. 433.

SILVEIRA,A.P.DIAS.;AZEVEDO, I.C.; OLIVEIRA,E.; MELETTI,L.M.M. Crescimento de plantas micorrizadas de maracujazeiro sob influência da adubação fosfática e da matéria orgânica. In: CONGRESSO LATINO AMERICANO DE CIÊNCIA DO SOLO, 13., 1996, Águas de Lindóia. Anais... Sociedade LatinoAmericana de Ciência do Solo, 1996.3p. CD-ROM.

SOUZA, A. da S.; ZAIDAM, H. A.; SOUZA, F. V. D.; SILVA, K. M. da; PAZ, O. P. da. Protocolo de micropropagação de bananeira através de ápices caulinares. Cruz das Almas-BA: Embrapa-CNPMF, 1994. 2p. (Banana em Foco, 3).

TEDESCO, N.; CALDEIRAL, M.V.W.; SCHUMACHER, M. V. Influência do vermicomposto na produção de mudas de caroba (Jacaranda micrantha Chamisso). Revista Árvore, Viçosa, v.23, n.1, p.1-8, jan./ mar. 1999.

TRINDADE, A.V.; SIQUEIRA, J.O.; ALMEIDA, F.P. Eficiência simbiótica de fungos micorrízicos arbusculares em solo não fumigado, para o mamoeiro. Revista Brasileira de Ciência do Solo, Viçosa, v. 24, n. 3 , p.505-513, 2000a.

TRINDADE, A.V.; FARIAS, N.G.; ALMEIDA, F.P. Uso de esterco no desenvolvimento de mudas de mamoeiro colonizadas com fungos micorrízicos. Pesquisa Agropecuária Brasileira, Brasília, v. 35, n. 7, p. 1389-1394, 2000b.

VIEIRA, A.H.; RICCI, M. dos S.F.; RODRIGUES, V.G.S.; ROSSI, L.M.B. Efeito de diferentes substratos para produção de mudas de freijólouro Cordia alliodora (Ruiz \& Pav.) Oken. Porto Velho: EMBRAPACPAF, 1998. 12p. (Boletim de pesquisa, 25).

ZAMBOLIM, L.; REIS, M. A. dos; COSTA, L. M. da. Substratos para multiplicação de inóculo do fungo micorrízico vesículo-arbuscular Glomus etunicatum. Fitopatologia Brasileira. Brasília, v.17, n.1, p.2831, 1992. 